rev.relac.int.estrateg.segur.11(1):147-169,2016

\title{
EL ENFOQUE DEL DIÁLOGO CIVILIZACIONAL DESDE AMÉRICA LATINA*
}

\author{
Isaac Caro** \\ Isabel Rodríguez ${ }^{* * *}$
}

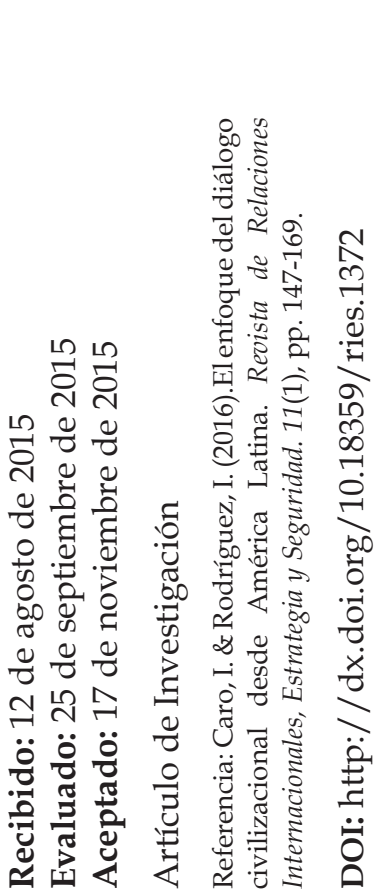

\section{RESUMEN}

Este artículo se enmarca en el debate teórico del paradigma del civilizacionismo en la disciplina de las relaciones internacionales, a partir de sus dos vertientes opuestas, la de choque de civilizaciones y la de diálogo de civilizaciones. El objetivo es demostrar su validez y aporte en la explicación teórica a problemas de la realidad internacional considerando tres niveles de análisis: internacional, regional, y local. En concreto, analizamos las propuestas de diálogo civilizacional y los encuentros de Alianza de Civilizaciones que tienen lugar en el marco de Naciones Unidas. Dicha tendencia global la explicaremos como problemática en América Latina tomando en consideración tres planes nacionales de Alianza

Este artículo forma parte del proyecto Fondecyt No. 1120401, "Conflicto y diálogo en Argentina y Chile: Los casos del judaísmo, el islam y el hinduismo en el periodo 2001 - 2011".

** Doctor en Estudios Americanos, mención en Relaciones Internacionales, Universidad de Santiago de Chile. Docente, Universidad Alberto Hurtado y Universidad Arturo Prat. Correo electrónico: icaro@uahurtado.cl

*** Doctora en Ciencia Política y Sociología, Universidad Complutense de Madrid. Docente, Universidad del Desarrollo, Santiago, Chile. Correo electrónico: isabelrodriguez@udd.cl 
de Civilizaciones, los de Argentina, Brasil y México, así como también analizaremos a nivel local ejemplos de diálogo institucionalizado que se registran en el caso argentino. Así, uno de los objetivos centrales de este artículo es establecer cómo se ha ido avanzando en una agenda de diálogo civilizacional en el ámbito de las relaciones internacionales, considerando algunos enfoques teóricos, así como prácticas de encuentros, foros y alianzas.

Palabras clave: América Latina, Diálogo Civilizacional, Relaciones Internacionales, Religión.

\section{THE PERSPECTIVE OF CIVILIZATIONAL DIALOGUE FROM LATIN AMERICA}

\section{ABSTRACT}

This article is part of a theoretical debate about the paradigm of civilizational dialogue that emerges in the discipline of International Relations as a new perspective of analysis as opposed to a focus on the clash of civilizations. The objective is to demonstrate its validity and contribution towards the theoretical explanation of problems in international reality by considering three levels of analysis: international, regional and local. In short, we analyse proposals of civilizational dialogue and Alliance of Civilizations encounters in the framework of the United Nations. We will explain this global trend as a Latin American problem, taking into consideration three National Plans for Civilizational Alliances: those of Argentina, Brazil and Mexico, with an additional analysis of local examples of institutionalized dialogue in the case of Argentina. Thus, one of the main objectives of this article is to establish how have been advanced in a civilizational dialogue agenda in the scope of the international relations, considering some theoretical approaches, thereby the practices of meetings, forums and alliances.

Keywords: Latin America, Civilizational Dialogue, International Relations, Religion.

\section{O ENFOQUE DO DIÁLOGO CIVILIZACIONAL DESDE AMÉRICA LATINA}

\section{RESUMO}

Este artigo centra-se no paradigma de civilizacionismo na disciplina de relações internacionais, com base em seus dois lados opostos, o choque de civilizações e o diálogo de civilizações. O objetivo é demonstrar a validade e a contribuição na explicação teórica para os problemas da realidade internacional, considerando três níveis de análise: internacionais, regionais e locais. Especificamente, analisamos as propostas de diálogo civilizacional e da Aliança de Civilizações, reuniões que têm lugar no âmbito das Nações Unidas. Esta tendência global explica problemas na América Latina, considerando três planos nacionais de Aliança de Civilizações, os da Argentina, Brasil e México, bem como localmente analisar exemplos de diálogo institucionalizado no caso argentino. Assim, um dos objetivos de este artigo é estabelecer como tem ido avançando em uma agenda do diálogo civilizacional no escopo das 
relações internacionais, considerando algumas aproximações teóricas, assim como práticas dos encontros, fóruns e alianças.

Palavras-chave: América Latina, Diálogo Civilizacional, Relações Internacionais, Religião.

\section{INTRODUCCIÓN}

Uno de los objetivos centrales de este artículo es establecer cómo se ha ido avanzando en una agenda de diálogo civilizacional en el ámbito de las relaciones internacionales, considerando algunos enfoques teóricos, así como prácticas de encuentros, foros y alianzas. En este sentido se considerará un nuevo paradigma, el del civilizacionismo, que subraya la importancia de los actores culturales y religiosos en las relaciones internacionales (Marchetti, 2009). Este paradigma tiene dos vertientes, una de choque o conflicto entre civilizaciones, otra de diálogo y entendimiento. Este civilizacionismo del diálogo es el enfoque que adoptaremos en el presente artículo, con la finalidad de evitar el reduccionismo de Huntington y Lewis, que son los principales exponentes del choque civilizacional, y aportar con ello a una nueva perspectiva que comienza influir los estudios internacionales en estos temas (Merinero, 2002; Dallmayr, 2009; Katzenstein, 2009; Michael \& Petito, 2009). El diálogo de civilizaciones es una propuesta de la sociedad internacional que busca entendimiento entre culturas, pueblos y religiones distintas, reconociendo y respetando la importancia de la diversidad cultural y religiosa, considerando que esta diversidad se encuentra también al interior de estas civilizaciones que no son unidades monolíticas.

Este artículo tiene cinco objetivos. Primero, entregar algunos antecedentes teóricos del paradigma civilizacional. Segundo, reseñar el nuevo enfoque del civilizacionismo basado en el diálogo y su validez teórica en las relaciones internacionales. Tercero, examinar la realidad internacional que muestra la viabilidad explicativa de este paradigma en cuanto se trata de un proceso de construcción de una cultura de paz y diálogo en los niveles local, nacional, regional y global (Naciones Unidas, 2009). Cuarto, examinar el aporte desde América Latina al diálogo civilizacional a partir de los planes nacionales elaborados por Argentina, Brasil y México para este fin. Y quinto, analizar los avances en la instauración de un diálogo institucionalizado en el caso de Argentina, en cuanto presenta mayores avances entre las comunidades judías, musulmanas y cristianas de este país, algo reconocido por el propio Departamento de Estado de Estados Unidos (Departamento de Estado, 2004).

En la trayectoria formativa del paradigma del civilizacionismo desde los años 90, tenemos dos momentos que mencionar como relevantes: 2001 y 2004. Al respecto, el año 2001 es un hito relevante tanto para la perspectiva del choque como para la vertiente dialógica, paradojalmente con dos sucesos fundamentales: los atentados del 11 de septiembre (11-S), y la consideración de aquel como el "Año de las Naciones Unidas para el Diálogo entre las Civilizaciones", idea propuesta desde 1997 por el entonces presidente de Irán, Muhammad Jatami, que a diferencia 
de otros mandatarios iraníes, se caracterizó por la búsqueda de una cercanía con Occidente y por enfatizar la necesidad de un diálogo, donde Irán fuera "el punto de encuentro de las culturas orientales y occidentales" (Jatami, 1998).

Por otra parte, el año 2004, muestra también algunos hitos importantes a considerar en nuestro análisis porque, como resultado de los atentados de Madrid, el gobierno español propuso en la Asamblea General de la ONU una Alianza de Civilizaciones (AC), esto es un encuentro entre occidente y el mundo árabe y musulmán, de modo de combatir el terrorismo por medios no militares. Y para el caso de América Latina, y Argentina en particular, existe un hito anterior, que se remonta a 1994, cuando se produce el atentado contra la Asociación Mutual Israelita Argentina (AMIA), lo que da origen a un diálogo institucionalizado entre comunidades judías, árabes, musulmanas y cristianas.

En consecuencia, analizaremos los efectos de estos hitos en la realidad internacional, considerando los niveles global, regional y local. A nivel global, analizaremos tres procesos que muestran la viabilidad explicativa de este paradigma en el marco de Naciones Unidas. El primero, la propuesta del presidente iraní, Mohamed Jatami, el año 1997, en la Cumbre de la Conferencia Islámica, proponiendo un diálogo de civilizaciones, lo que se concreta el año 2001, cuando la Organización de las Naciones Unidas (ONU) proclama el "Año de las Naciones Unidas para el diálogo de la Civilizaciones". El segundo, liderado por la ONU para la Educación, la Ciencia y la Cultura (UNESCO), con base al concepto de diálogo interreligioso en el marco del diálogo intercultural, concretado en noviembre de 2001, con la "Declaración Universal sobre Diversidad Cultural". El tercero, los encuentros sobre AC que se originan en el año 2004 bajo la iniciativa de los gobiernos de España y Turquía, y que hasta el año 2013 ha dado origen a cinco foros internacionales.

A nivel regional, consideraremos América Latina, tomando como referencia tres planes nacionales de la AC que son los de Argentina, Brasil y México, y que se caracterizan por enfatizar cuatro áreas principales de aplicación para fomentar el diálogo: educación, migraciones, jóvenes, y medios de comunicación. Por último, a nivel local, analizaremos el caso argentino que adquiere un carácter emblemático dadas algunas condiciones específicas de este país sudamericano: primero, en él se encuentra la comunidad judía más numerosa e importante de la región; segundo, tienen lugar dos atentados considerados los más graves realizados en toda la región, y uno de ellos, el atentado más importante en contra de un colectivo judío desde terminada la Segunda Guerra Mundial; y tercero, se han desarrollado instancias de diálogo entre las comunidades judías y musulmanas apoyadas por la Iglesia Católica, y lideradas por el entonces cardenal Bergoglio, actual Papa Francisco.

En este artículo buscamos demostrar que existe una propuesta de diálogo civilizacional en el ámbito de las relaciones internacionales, la que, superando el paradigma estatocéntrico, concibe la importancia de la religión y la cultura en el sistema internacional. Esta propuesta está compuesta de enfoques teóricos, así como prácticas de encuentros, foros y alianzas, 
las que operan a nivel global (Naciones Unidas, Unesco, AC), regional (estrategia regional latinoamericana, planes nacionales de AC) y local (caso argentino de diálogo entre comunidades judías, árabes y musulmanas, con un rol central de la Iglesia Católica).

El análisis se divide en cinco partes. En la primera, se consideran algunos antecedentes del paradigma civilizacional, especialmente en su versión de choque. En la segunda se examina el enfoque del civilizacionismo en su corriente dialógica, especialmente los aspectos teóricos con los que aporta a la disciplina de las relaciones internacionales. En la tercera parte, se estudian algunos casos emblemáticos de encuentros y foros civilizacionales como instancias de diálogo desarrolladas por la sociedad internacional. En la cuarta parte, se reseñan algunos de los aspectos centrales de los planes nacionales de Argentina, Brasil y México para impulsar el diálogo civilizacional. Y finalmente, en la quinta parte, analizamos los avances del caso argentino en institucionalizar este diálogo entre las comunidades judías, musulmanas y católicas en este país.

\section{Algunos antecedentes del paradigma civilizacional}

El civilizacionismo da un espacio central a las civilizaciones y a los actores religiosos, importantes del sistema internacional. Está centrado en la primacía del vínculo cultural, reconociendo los aspectos culturales y religiosos de la vida humana como predominantes, y hace referencia primariamente a las civilizaciones y élites culturales como actores claves en el sistema internacional (Marchetti, 2009). En consecuencia, el concepto de civilización es central a este enfoque, considerado como una unidad macro social y macro política, que, en su uso plural "civilizaciones", se entiende como una visión de mundo, incluyendo costumbres, estructuras y cultura, que constituyen un todo histórico que coexiste con otras civilizaciones; a diferencia del uso singular, donde "civilización" está en oposición a "barbarie" (Wallerstein, 1997; Salter, 2002; Katzenstein, 2009). Siguiendo a estos autores, sostenemos que hoy día la civilización es un concepto clave para una interpretación de la política global, más allá de una perspectiva basada y limitada en el Estado. Su valor reside en su fuerte acento en los componentes culturales y religiosos de los asuntos internacionales y su criticismo de la globalización neoliberal.

Este paradigma tiene un antecedente destacado para las relaciones internacionales en el contexto europeo de la Primera Guerra Mundial, cuando el filósofo alemán Oswald Spengler publicó La Decadencia de Occidente (1918), para referirse a la involución que caracteriza al mundo occidental y, a Europa en particular, coincidiendo con el estallido de la Gran Guerra. Luego, resurge con fuerza en el contexto posguerra fría, cuando en el marco de los nuevos conflictos internacionales algunos académicos, especialmente estadounidenses, enarbolaron nuevas "teorías" para explicar el escenario que surgía tras la desintegración de la Unión Soviética.

Estas explicaciones (Lewis, 1990; Huntington, 1993, 1996) postulaban que el enfrentamiento ideológico entre comunismo y capitalismo, entre Estados Unidos y la Unión Soviética, sería sustituido por un choque civilizacional entre Occidente-constituido por Norteamérica y Europay muchas civilizaciones no occidentales, siendo una de las más importantes la civilización 
islámica. Bernard Lewis (1990), en un artículo titulado "Las raíces del odio musulmán", da cuenta de una lucha histórica entre el islam y el cristianismo que se inicia desde los momentos mismos de la fundación de aquél en el siglo VII y que persiste hasta nuestros días. Más adelante, Samuel Huntington (1993) publica su famoso artículo titulado "¿Choque de civilizaciones?", donde da cuenta de un conflicto entre occidente y los Estados confucianos e islámicos a partir de una conexión militar confuciana - islámica, diseñada para promover la adquisición de armas y tecnología bélica necesarias para contrarrestar el poder militar occidental. Posteriormente, Huntington (1996) elabora más minuciosamente un "paradigma civilizacional" que da cabida al enfrentamiento entre civilizaciones, donde el denominado "Resurgimiento islámico" tiene un papel importante.

Las críticas al choque civilizacional proceden de distintos autores. Mark Salter (2002) sostiene que Huntington ha promovido la imagen de que cada musulmán es un potencial terrorista, y que los bárbaros y los musulmanes son los potenciales enemigos naturales de la civilización. El autor se concentra en el discurso dicotómico europeo occidental sobre la civilización y la barbarie, considerando la importancia de este discurso en las relaciones internacionales: la resurrección del choque civilizacional representa un nuevo "realismo civilizacional", el que reinscribe el "orden imperial" en la posguerra fría.

Para Fukuyama (2001), el conflicto actual no es parte de un choque de civilizaciones; "más bien, es sintomático de una acción de retaguardia por parte de quienes se sienten amenazados por la modernización. Said (2002) señala que "no son 'civilizaciones' las que se enfrentan en el actual conflicto" (p.221), puesto que tanto occidente como el islam no pueden considerarse identidades cerradas. $Y$ el islam ya no está en la periferia de occidente sino en su centro. Para Ferguson (2006), más que un choque de civilizaciones, lo que existe son "civilizaciones chocadas", esto es conflictos que tienen lugar al interior de una misma civilización, lo que queda demostrado en el hecho que desde el fin de la Guerra Fría han proliferado guerras civiles o enfrentamientos al interior de una misma unidad cultural o civilizacional. Según Sen (2006), la tesis del choque de civilizaciones tiene dificultades porque se da por supuesta una única clasificación, de modo que la pregunta ¿chocan las civilizaciones? se funda bajo el supuesto de que la humanidad puede ser agrupada en civilizaciones concretas.

En consecuencia, el choque civilizacional lleva a un enfrentamiento entre el bien y el mal, en donde el enemigo, representado por el islam, resulta incompatible con la modernidad y la democracia (Fernández, 2008). Al igual que el 11-S, los atentados del 12 de octubre de 2002 en Bali, del 11 de marzo de 2004 (11-M) en Madrid, del 7 de julio de 2005 en Londres, del 26-29 de noviembre de 2008 en Bombay, entre otros, han avivado un debate sobre los "peligros" que representa el islam, resaltando la dinámica de guerra civilizacional, al tiempo que han sido un factor importante en los aumentos de la islamofobia que se han registrado en el mundo (International Helsinki Federation for Human Rights, 2005). En este sentido, la islamofobia, entendida como situaciones de rechazo hacia el islam y los musulmanes, donde estos son considerados como "bárbaros", parece ser resultado en parte importante de las 
"teorías civilizacionales" y consiguientes políticas implementadas por Estados Unidos hacia el islam, las que se profundizan a la luz de los atentados del 11-S.

Sostenemos que el paradigma de choque civilizacional tiende a promover generalizaciones, donde la visión de las civilizaciones como unidades monolíticas no considera las divisiones que se dan al interior del islam, del judaísmo, del hinduismo o de occidente. Hay que considerar que existen factores empíricos que muestran las pocas probabilidades de un choque entre el islam y occidente, en cuanto aquel no es una unidad homogénea, que dice relación en primer lugar, con la diversidad y complejidad interna del mundo islámico; segundo, con una trayectoria histórica diferenciada de los países árabes con respecto al resto del islam; y tercero, con un proceso hacia un aumento del modo de vida urbano industrial (López, 2002). Además, existe una tendencia a que persistan y se consoliden conflictos al interior del islam, no sólo entre sectores chiitas y sunitas (Irak, Líbano, Siria, Bahréin), sino también entre posiciones laicas y religiosas (Egipto, Siria, Palestina).

Aunque es indiscutible que el conflicto y la guerra persisten en la sociedad internacional de la posguerra fría, ellos adquieren nuevas características y dimensiones, siendo el paradigma civilizacional, en particular en su vertiente del choque, incapaz de dar cuenta de toda su complejidad. En este sentido, nos parece importante mencionar los enfoques que subrayan la incorporación de nuevas formas de guerra que se generan con los cambios en la posguerra fría, así como con la consecuente erosión y transformación de los Estados nacionales. Se trata específicamente de las "guerras del futuro" a partir de las experiencias de Ruanda 1990-1993, Somalia 1991, Bosnia 1992-1995 (Holsti, 1996); de las "guerras posmodernas", donde son centrales las ciberguerras, así como los usos de la ciencia y la computación como fue mostrado en la guerra de Kuwait en 1991 (Gray, 1997); de las "nuevas guerras", que constituyen reivindicaciones identitarias, violaciones a los derechos humanos, violencia organizada y dirigida en contra de la población civil en consonancia con las tácticas de la contrainsurgencia y de la limpieza étnica (Kaldor, 2001, 2006); de las "guerras del siglo XXI", que implican cambios en las relaciones políticas, el progreso tecnológico y el cambio cultural, lo que hace que estas guerras se caractericen por una interdependencia entre violencia elemental, creatividad estratégica y racionalidad política (Münkler, 2003); o bien de guerras internas y civiles a escala global, que representan una amenaza tanto a la seguridad como al desarrollo (Collier, 2004; Duffield, 2004).

También podemos mencionar los enfoques que se oponen a las tesis de las "nuevas guerras", al señalar que el impacto humano del conflicto civil es más bajo en el periodo de la posguerra fría, puesto que existe una caída del conflicto ideológico así como una menor influencia de la globalización sobre los gobiernos (Melander, et al. 2006); se trata de "nuevas guerras civiles", que constituyen fenómenos criminales más que políticos (Kalyvas, 2001). Existen muchas otras fuentes sobre el conflicto y la guerra, algunas de ellas procedentes de enfoques estructuralistas, que profundizan en aspectos particulares sobre guerra civil, ghetto y prisión (Wacquant, 2000) o sobre la relación entre terrorismo y violencia (Borradori, 2003). 
En consecuencia, este artículo no sostiene que el enfoque del diálogo civilizacional pueda erradicar el conflicto de las relaciones internacionales en cuanto muchos se encuentran activos, pero si negamos las premisas que consideran que la diferencia entre civilizaciones es determinante como causa del conflicto. En efecto, el enfoque del diálogo releva otros aspectos y factores que pueden explicar las dinámicas de cooperación y diálogo en el sistema internacional y lo más importante lograr institucionalizarlas.

\section{El paradigma del diálogo de civilizaciones}

En oposición al enfoque del choque de civilizaciones que hemos analizado anteriormente, en el contexto de posguerra fría emerge tímidamente un discurso sobre el diálogo de civilizaciones en las relaciones internacionales, que se opone a la concepción monolítica del discurso bipolar de dos potencias enfrentadas, el que coloca énfasis en categorías explicativas para el nuevo escenario como son paz, cambio global, cooperación, diálogo y multilateralismo. Este enfoque comienza a generar una línea teórica y de análisis que se consolida a través de un aumento de literatura relativa a partir del año 2001, y que tiene dos ejes principales. En primer lugar, y como ya se ha explicado, el enfoque del diálogo civilizacional se construye a partir de desarrollos críticos hacia la visión de choque civilizacional, ya sea enfatizando el carácter eurocéntrico de la visión de Huntington o bien señalando que los conflictos al interior de las civilizaciones tienen más evidencias empíricas que los que se desarrollan entre civilizaciones distintas. En segundo lugar, se desarrolla un enfoque propio a partir de una propuesta de diálogo civilizacional, que enfatiza la importancia de nuevas identidades, culturales, religiosas, civilizacionales, en el periodo de posguerra fría a partir de una crítica al realismo político y al discurso bipolar característico de la Guerra Fría. En tercer lugar, estos enfoques se relacionan con una práctica que consiste en encuentros, foros sobre diálogo y, particularmente, la iniciativa de AC, que lleva a ciertas actividades institucionalizadas como se veremos.

Uno de los principales representantes de esta línea es Fred Dallmayr (2002), quien señala que las dificultades del diálogo civilizacional se dan con particular referencia a Europa y a la cultura occidental en su relación con el contexto global no occidental. Aquí, las memorias del colonialismo y el imperialismo, junto con los traumas de la conquista y el genocidio, han inducido a muchos observadores a un cansancio con Europa, una tendencia a depreciar la cultura occidental/europea como sinónimo de arrogancia cultural. Como un modo de superar esto, se deben delinear las estructuras y precondiciones del diálogo civilizacional, lo que presupone cierta igualdad cívica entre los participantes, y superar las divisiones entre el norte y el sur, entre el mundo desarrollado y el subdesarrollado.

Según Dallmayr, existen tres modalidades o formas de diálogo, las que tienen diferentes expresiones en las relaciones internacionales (2002). La primera modalidad, comunicación pragmática-utilitaria, es cercana al dominio del monólogo, donde cada actor busca primariamente sus propios intereses, sus propias metas y agendas. Este tipo de comunicación forma la base de la economía moderna y de la teoría de la elección racional, y tiene un 
rol en las relaciones políticas e internacionales, a través de la escuela realista, el énfasis en el interés nacional, la diplomacia tradicional. La segunda modalidad corresponde al discurso moral-universal, cuyo objetivo es establecer reglas generales y universales o normas de conducta válidas para todos los participantes en una interacción dada. Este discurso se encuentra en la filosofía moral de Kant, en el moderno derecho natural, en las reglas del derecho internacional (desde el derecho de gentes y la jurisprudencia española hasta Hugo Grocio); también está presente en las normas sobre crímenes de guerra, la Convención de Ginebra, la Declaración Universal de Derechos Humanos. La tercera modalidad es el diálogo ético-hermenéutico, en donde cada actor busca entender y apreciar las historias de vida y antecedentes culturales del otro, incluyendo sus tradiciones religiosas o espirituales. Algunos ejemplos son los diálogos interreligiosos, el Parlamento de las Religiones Mundiales, el Foro Social Mundial, el Foro Público Mundial. A partir de los niveles anteriores, Dallmayr señala que el término contrario a diálogo es "monólogo", que en el contexto político internacional corresponde a una política de unilateralismo o a una situación donde un poder hegemónico o imperial reduce a todos los otros agentes al silencio. Por el contrario, el diálogo, ya sea en su segunda o tercera modalidad, se traduce políticamente en una política de cooperación multilateral (Dallmayr, 2002; 2009).

La posición dialógica de Dallmayr se complementa con la concepción de H. Gadamer sobre la "unidad en la diversidad" y el carácter reflexivo y autocrítico del pensamiento europeo, la crítica al eurocentrismo y al pensamiento filosófico europeo desarrollada por E. Dussel, la visión de la no violencia activa planteada por M. Gandhi, la concepción de libertad pública de $\mathrm{H}$. Arendt, la crítica de la libertad negativa de C. Taylor, la visión sobre justicia y democracia de I. M. Young (Dallmayr, 2002).

Otros autores, como Hall y Jackson abordan los encuentros entre civilizaciones a partir de tres factores principales: estándares globales de la civilización de mercado; deconstrucción del eurocéntrico choque de civilizaciones; status de la mujer en los estadios civilizacionales. Ambos autores se enfocan en aquellas prácticas sociales y políticas a través de las cuales las nociones de identidad civilizacional son reproducidas en una variedad de contextos, desde el régimen global a debates teológicos sobre la modernidad y la guerra contra el terrorismo, a partir de lo cual enfatizan en la necesidad de un diálogo entre distintas civilizaciones (Hall \& Jackson, 2007). Por su parte, el intelectual suizo de origen musulmán, Tarik Ramadan, señala que es importante un mayor entendimiento y educación sobre nuestra historia, cultura y religión, valores que deben ser transmitidos a otras culturas, religiones y visiones de mundo. Defiende un pluralismo abierto al mundo, a comunicarse y a aprender a respetar las sensibilidades y opiniones de otras sociedades y culturas como principal requisito para un diálogo civilizacional (Ramadan, 2011).

Michális Michael y Fabio Petito, sostienen que terminado el sistema bipolar, emergió el "diálogo de civilizaciones" para oponerse al "monólogo del Imperio", que representaba el 
discurso bipolar de dos potencias enfrentadas. Agregan que, aunque varias organizaciones internacionales y jefes de Estado han promovido la idea de un diálogo entre civilizaciones, éste no ha prosperado porque tiende a prevalecer una matriz de etnocentrismo occidental, relacionado con el paradigma de choque de civilizaciones, en el discurso académico sobre la política y las relaciones internacionales. Igualmente consideran que el diálogo de civilizaciones se construye teóricamente en contra del monólogo, dominado ideológicamente por el mundo liberal y centrado en occidente y Estados Unidos. Se trata de la búsqueda de una nueva "unidad en la diversidad" para defender la pluralidad y el multilateralismo, impidiendo así la hegemonía de cualquier potencia en la política mundial (Michael \& Petito, 2009). En esta misma línea, Petito señala que el discurso político del diálogo de civilizaciones llama para una rediscusión de los argumentos liberales, centrados en Occidente, sobre los cuales la sociedad contemporánea internacional está basada (Petito, 2009).

Uno de los principales argumentos de los defensores del diálogo de civilizaciones es una crítica normativa (idealista) al discurso realista del poder político con tres dimensiones centrales: a) multipolaridad, con la emergencia de un orden mundial más pluralista y descentralización del poder hacia otros poderes regionales, como China, India, Japón, Rusia, Brasil, Irán; b) nuevo derecho de gentes intercultural, lo que implica la emergencia de una sociedad internacional multicultural, cosmopolita; c) la paz como un objetivo del diálogo de civilizaciones, buscando construir puentes, lo que puede estar ejemplificado en Turquía como puente entre Asia y Europa, así como en las iniciativas del Mediterráneo que en la década de 1990 incorporaron a países europeos y árabes (Petito, 2009), además de Turquía e Israel y de cuya inspiración nace la AC como se verá más adelante. Al mismo tiempo, el siglo XX evidenció iniciativas innovativas en la arena política para establecer estructuras internacionales compartidas: la Liga de Naciones, las Naciones Unidas, la formación de la Unión Europea. En una escala más limitada, hay iniciativas similares de integración en Asia, África y América Latina. En el mundo musulmán, la idea de umma (comunidad de todos los musulmanes) ha ganado adhesión como alternativa al Estado nación (Dallmayr, 2009), que encuentra un referente en la Organización para la Cooperación Islámica, una entidad macro nacional formada en 1969 donde tienen representación los Estados de confesión musulmana.

En definitiva, el discurso político que erige el diálogo de civilizaciones llama a la reapertura y rediscusión de los argumentos liberales y realistas en los cuales la sociedad contemporánea internacional está basada, intentando colocar en la agenda internacional temas como cooperación, diversidad, multiculturalismo, paz global y derechos humanos. Su aporte está en la mirada cultural y civilizacional, a partir de una nueva perspectiva que da importancia a los actores no estatales y nuevas categorías de análisis, centradas fundamentalmente en el diálogo y entendimiento entre pueblos, culturas y civilizaciones distintas. En este sentido, es indiscutible que una contribución fundamental ha sido la propuesta de una $\mathrm{AC}$, la que se ha concretado e institucionalizado a través de una serie de mecanismos, que se verán en el apartado siguiente. 


\section{El nuevo paradigma: encuentros, foros de diálogo y la alianza de civilizaciones}

En este acápite analizaremos la realidad internacional que muestra la viabilidad explicativa del paradigma civilizacional en su vertiente dialógica, demostrando los efectos de los sucesos que acontecen alrededor de dos hitos de la realidad internacional, 2001 y 2004, en cuanto impulsadores de las dinámicas de institucionalización de diálogo civilizacional a nivel internacional.

La necesidad de crear foros institucionalizados como la AC tiene sus antecedentes en la década de los 90, a partir de la Conferencia de Paz de Madrid de 1991, y de la Declaración de Barcelona de 1995. La Conferencia de Madrid, convocada conjuntamente por Estados Unidos y la Unión Soviética, inauguró negociaciones bilaterales de Israel con Jordania, Siria, Líbano y la Organización de Liberación de Palestina (OLP), además de negociaciones multilaterales, en donde estaban representados los principales actores mundiales, Estados Unidos, Rusia, Japón, China y la Unión Europea. En un contexto de acercamiento entre Israel y el mundo árabe (concretado en la Declaración de Principios con la OLP en 1993, y en el Tratado de Paz entre Israel y Jordania en 1994), la Unión Europea junto a Turquía, Israel, la Autoridad Palestina y seis países del mundo árabe (Egipto, Jordania, Líbano, Marruecos, Siria, Túnez), suscribieron en noviembre de 1995 la llamada "Declaración de Barcelona". El objetivo era ambicioso: hacer del Mediterráneo un espacio común de paz, mediante el diálogo político y de seguridad, la creación de una zona de libre comercio, la promoción de una asociación cultural a través del diálogo intercultural e interreligioso (Conferencia euro-mediterránea, 1995). Sin embargo, el bloqueo en las negociaciones con los palestinos hizo imposible continuar el diálogo entre los países de las dos orillas del Mediterráneo. La presencia de Israel en los foros era cuestionada por los países árabes, a excepción de Egipto y Jordania, que eran los únicos que mantenían tratados de paz con el Estado judío. El fracaso de Madrid y Barcelona era evidente.

Posteriormente, en la Cumbre de la Conferencia Islámica, realizada en Teherán el año 1997, el entonces presidente iraní, el reformista Mohamed Jatami, propuso establecer un año para el "diálogo de civilizaciones", idea que fue rescatada por las Naciones Unidas para considerar el 2001 como el "Año de las Naciones Unidas para el Diálogo de las Civilizaciones". De manera enfática, Jatami señaló que para que exista un verdadero diálogo entre civilizaciones resulta indispensable que oriente "se convierta en un participante real" y que no sea solo un "objeto de estudio". Este es un paso que debe dar Europa, Norteamérica y también el islam para obtener un verdadero conocimiento de occidente y de oriente (Jatami, 1998).

Siguiendo con la posición del diálogo de civilizaciones, la Asamblea General de la UNESCO, celebrada en París, entre octubre y noviembre del 2001, en el marco del Programa Mundial para el Diálogo entre Civilizaciones (Resolución 56/6), aprobó la "Declaración Universal sobre Diversidad Cultural", a partir de la necesidad de profundizar el debate internacional sobre los problemas relativos a la diversidad cultural, lo que implica un compromiso en el campo 
del diálogo entre civilizaciones (UNESCO, 2002). Por su parte, la Conferencia Ministerial Internacional de Diálogo entre Civilizaciones, realizada en Nueva Delhi, en julio de 2003, discutió la necesidad de fortalecer la diversidad religiosa, como parte de la diversidad cultural (Fuentes, 2005).

Después de los atentados de marzo de 2004 en España, se promueve y valida en Naciones Unidas la necesidad de crear foros institucionalizados como la AC. Es así como en septiembre del mismo año, en el marco de la Asamblea General de la ONU, el presidente español, José Rodríguez Zapatero, propuso una AC colocando como referente del mismo el diálogo euromediterráneo iniciado en Barcelona el año 1995, que había reunido a los Estados europeos del Mediterráneo occidental y a los países árabes del Mediterráneo oriental, además de Turquía e Israel. La nueva iniciativa fue firmemente respaldada por Turquía y su primer ministro, Recep Erdogan, dando origen a los denominados "Foros de Alianza de Civilizaciones", que han contado con el patrocinio de los gobiernos de Madrid y de Ankara.

Entre 2007 y 2013 se realizaron cinco Foros de AC (Madrid 2007, Estambul 2009, Río de Janeiro 2010, Doha 2011, Viena 2013) creando una institucionalización del diálogo en la sociedad internacional. Los principales componentes de esta institucionalización son: a) los planes nacionales para la AC, con la finalidad de llevar a cabo acciones específicas por parte de cada Estado para el cumplimiento de los objetivos de la Alianza; b) la conformación de un Grupo de Amigos de la AC, con119 países (incluyendo Estados Unidos, Rusia, China, la mayoría de los Estados europeos y todos los sudamericanos a excepción de Venezuela) y 22 organizaciones, entre ellas la Liga de Estados Árabes, la OEA y la Unión Africana; c) la creación de un Instituto Internacional de la AC integrado en la Universidad de las Naciones Unidas, financiado por el gobierno español y la Unión Europea, que tiene como principales áreas de trabajo la educación, las migraciones y rol de los medios de comunicación; d) la conformación de estrategias regionales que tienen por objetivos que cada región pueda aportar a los objetivos de la Alianza, existiendo tres estrategias, la del sudeste europeo, creada en 2009; la del Mediterráneo, establecida en 2010; y la de América Latina, formada en febrero de 2013.

Además de las instancias anteriores, que tienen por finalidad hacer partícipes a toda la comunidad internacional, se han creado una gran cantidad de centros que buscan fortalecer el diálogo cultural, religioso y civilizacional. Algunos ejemplos: Centro Internacional de Toledo para la Paz y Centro Malaika en España; Tony Blair Foundation en Gran Bretaña; Centre for Dialogue, de La Trobe University, en Australia. Resulta emblemática la Fundación BarenboimSaid, que lleva los nombres del músico judío argentino Daniel Barenboim y del intelectual palestino Edward Said, quienes formaron juntos una orquesta con jóvenes judíos y palestinos, que ha recorrido gran parte del mundo. Hay que mencionar también la Fundación para el Diálogo entre Civilizaciones, creada y dirigida por el ex presidente iraní Jatami en 2007, cuya propuesta inicial sobre diálogo expuesta en 1997 cobró renovada fuerza después del acuerdo alcanzado en 2013 por el nuevo régimen iraní, conducido por el presidente Hassan Rouhani, con las seis potencias Estados Unidos, Rusia, China, Reino Unido, Francia y Alemania, en 
relación al programa nuclear iraní. La Fundación está estrechamente relacionada con cuatro organizaciones preocupadas por el diálogo, la paz y los derechos humanos: el Centro de Oslo para la Paz y los Derechos Humanos, establecido en 2006; la Catedral Nacional de Washington, cuya construcción se terminó en 1990 y es conocida porque allí se realizan ceremonias de todas las religiones; la Fundación de Cultura para la Paz, creada en 2000 por la Comunidad de Madrid; el Club de Madrid, fundación establecida en 2004, cuya función principal es trabajar en la resolución de conflictos.

En conclusión, hay un avance importante en la institucionalización del diálogo de civilizaciones en el marco de organizaciones internacionales amparadas en Naciones Unidas. Sin embargo, los obstáculos son muchos, principalmente actores y procesos internacionales que se sustentan y legitiman aumentando el nivel de conflictos, como por ejemplo, los grupos jihadistas del Medio Oriente y África, la espiral de violencia entre Israel y Hamas, los enfrentamientos entre Ucrania y sectores separatistas pro-rusos, siendo estos fenómenos, entre muchos otros, un obstáculo a la AC.

\section{América Latina y la alianza de civilizaciones}

En el ámbito latinoamericano, hay que destacar la presencia de una estrategia regional de AC que ha contado con la participación de 14 países (México, todos los Estados sudamericanos salvo Venezuela, y Costa Rica, El Salvador, Guatemala y Nicaragua), centrada en cuatro grandes tópicos: educación, jóvenes, migraciones, medios de comunicación. Esta estrategia surge a partir del tercer foro, realizado en Río de Janeiro en 2010, y tiene el objetivo de fomentar de manera regional los principios de la Alianza que se impulsan en Naciones Unidas, lo que ha dado origen a los planes nacionales de México, Brasil y Argentina.

A nivel regional hay que mencionar también las tres reuniones cumbres de América del Sur y los Países Árabes (ASPA), que han tenido lugar en Brasilia (2005), Qatar (2009) y Lima (2012), las que tienen por finalidad la cooperación en los ámbitos de la economía, la cultura, la educación, la ciencia y la tecnología, así como la promoción de la paz mundial. Algunas iniciativas concretas de cooperación son las siguientes: creación de una biblioteca árabe-sudamericana, lucha en contra de la desertificación, fortalecimiento del intercambio de información y tecnología, implementación de políticas para superar la pobreza y la desigualdad. En la Declaración de Lima de 2012 se enfatizó de manera explícita la relación entre estas cumbres y la AC como uno de los objetivos principales:

Reafirmar la importancia de ASPA como un mecanismo de cooperación birregional y diálogo intercultural. Recordar, en este sentido, las acciones relevantes desarrolladas para mejorar el conocimiento mutuo de las culturas de ambas regiones, en plena conformidad con los principios y objetivos de las Naciones Unidas establecidos en la III y IV sesión del Foro de la Alianza de Civilizaciones, llevadas a cabo en Río de Janeiro en mayo de 2010 y en Doha en diciembre de 2011, respectivamente, y otros foros relacionados. (ASPA, 2012) 
Además, existen en la región tres planes nacionales sobre AC, los de México, Brasil y Argentina. El plan de México, el que es más declaratorio y propositivo, destaca que éste ha sido un activo promotor de la paz, buscando en el marco de la ONU que se implementen iniciativas para el mejoramiento de la vida de las personas en el Medio Oriente, impulsando el diálogo para la solución integral de los conflictos en esa región. Al mismo tiempo, se señala que México tiene un rol importante en la AC debido a su carácter multicultural, que se sustenta por los pueblos y comunidades indígenas que se "entrelazan con el desarrollo de una sociedad moderna y cada día más compleja, en la que conviven y crecen culturas diversas que son iguales en derechos" (Secretaría de Relaciones Exteriores de México, 2010) .

El Plan Nacional de Brasil por su parte, destaca que este país ha forjado en su propio territorio una suerte de $\mathrm{AC}$, que es resultado de la contribución de diferentes grupos étnicos, y también de la integración de inmigrantes de muchas culturas y regiones del mundo. En lo que se refiere a política exterior, el país ha promovido cuatro grandes iniciativas que buscan el acercamiento con otros países y continentes: a) el Foro Trilateral IBSA, que incluye a India, Brasil y Sudáfrica; b) las cumbres ASPA, que reúne a los jefes de Estado y gobierno de Sudamérica y los Estados Árabes; c) las cumbres ASA, entre países de Sudamérica y África; d) las reuniones de la Comunidad de Países de Lengua Portuguesa (CPLP) (Ministério das Relações Exteriores, 2009).

El Plan Nacional argentino, al igual que los planes nacionales desarrollados por los respectivos Ministerios de Relaciones Exteriores de Brasil y México, resalta el énfasis que la comunidad internacional y la AC, en general, y Argentina, en particular, deben colocar para aumentar el entendimiento y la cooperación entre las sociedades occidentales y musulmanas, evitando de este modo la islamofobia, la xenofobia y las distintas formas de intolerancia. En lo concreto, este plan, lo mismo que las otras iniciativas latinoamericanas, trabaja cuatro grandes ámbitos: educación, jóvenes, migraciones y medios de comunicación.

Detallando el caso de Argentina, el plan busca, en materia de educación, permitir la integración de alumnos/as provenientes de distintos orígenes, así como desarticular prácticas discriminatorias. Especial importancia adquiere la participación del Instituto Nacional contra la Discriminación, la Xenofobia y el Racismo (INADI), así como la incorporación del estudio del holocausto en los planes curriculares. En el tema de jóvenes, se reconoce a estos como fuente de movilización y actores autónomos, fortaleciendo los espacios de inclusión, participación y comunicación, con el fin de producir un "diálogo cultural significativo". En lo referente a migraciones, se toma como referencia la Declaración de Santiago sobre Principios Migratorios (2004), cuyo objetivo es que cualquier persona nacida en un país del bloque pueda obtener una residencia regular en otro país que lo integre. Y en materia de medios de comunicación, se propone favorecer la participación de la comunidad para evitar las distintas formas de discriminación (Ministerio de Relaciones Exteriores de la República Argentina, 2010).

También es importante mencionar para la región el caso de Chile, que si bien no cuenta con un Plan Nacional al respecto, inauguró en 2007 en Coquimbo el Centro Mohammed VI para el 
Diálogo de Civilizaciones como una iniciativa del Reino de Marruecos, lo que ha dado origen a cinco encuentros internacionales sobre diálogo, realizados en las ciudades de Santiago, Coquimbo y Viña del Mar.

En conclusión, el enfoque de diálogo civilizacional nos muestra que, desde América Latina emergen iniciativas que se inscriben en los acuerdos, en el reconocimiento mutuo y que pueden, de este modo, contribuir a una AC a través de las experiencias regionales propias que implican el multilateralismo, la cooperación internacional, la lucha en contra de la discriminación, la defensa de la diversidad, entre muchos otros temas. No obstante lo anterior, se hace necesario un rol más protagónico de los demás países de la región, además de Argentina, Brasil y México, en sus compromisos con la AC. Queda como un tema pendiente la elaboración de nuevo planes nacionales, que incorporen oficialmente los temas de diálogo en las agendas políticas y de relaciones exteriores de los respectivos países latinoamericanos, siguiendo los ejemplos anteriores. En especial Chile tiene un rol importante que cumplir en el diálogo cultural y religioso, puesto que en el país se encuentra la comunidad palestina más grande existente en la diáspora, así como una comunidad judía, ambas incorporadas a las distintas actividades políticas, económicas, sociales y culturales de la vida nacional. Aunque tradicionalmente han existido buenas relaciones (personales, económicas, afectivas) entre judíos y palestinos, los vínculos formales entre ambas comunidades se han deteriorado a partir de la segunda intifada de septiembre de 2000.

\section{El caso de Argentina y los avances del diálogo civilizacional}

Para el caso argentino, el desarrollo de un Plan Nacional de AC es un imperativo sustancial, dadas las condiciones propias de su realidad nacional, que tienen relación con tres constataciones: en primer lugar, la diversidad religiosa y cultural del país, con una población musulmana estimada en 700.000 (Organización Islámica para América Latina, 2014) y la presencia de la comunidad judía más grande de la región latinoamericana, estimada en 182.000 (Della, 2012); segundo, el rol de la Iglesia Católica promoviendo iniciativas de diálogo, encabezadas por el cardenal Bergoglio, actual Papa Francisco; y tercero, la vivencia de situaciones de conflicto derivadas de los atentados de 1992 contra la Embajada de Israel y 1994 contra la AMIA, adjudicados, según pruebas judiciales argentinas, a Irán y al movimiento Hezbollah. Cabe señalar que este último atentado, que provocó 85 muertos, ha sido considerado el más grave realizado en contra de una comunidad judía después de la Segunda Guerra Mundial y el más grave de la historia argentina.

1. La investigación por la "Causa Amia" Ileva más de 20 años, sin que hasta la fecha existan personas detenidas. Judicialmente, se han seguido dos pistas: la denominada "pista internacional" que apunta a la culpabilidad de las más altas autoridades políticas y religiosas iraníes de la época, así como al movimiento Hezbollah; y la "pista nacional", referida a la obstrucción a la justicia por parte de personas encabezadas por el ex presidente Menem, así como el juez de la época, Juan José Galeano, representantes de los servicios de inteligencia argentina y de la propia comunidad judía argentina. La muerte del fiscal Alberto Nisman, en enero de 2015, quien investigaba esta causa, ha incorporado mayor complejidad al atentado realizado en 1994, incluyendo consecuencias políticas internas, así como interrogantes sobre las relaciones de Argentina con Irán. 
Efectivamente, Argentina es ejemplo paradigmático de diálogo institucional durante la década de 1990, que paradojalmente tiene un punto de inicio en el atentado contra la AMIA en 1994. Como consecuencia de este ataque, se inicia una ola importante de denuncias contra árabes y musulmanes, los que son acusados de terroristas, algo similar a lo que ocurre después del 11-S de 2001 en Estados Unidos o del 11-M de 2004 en España, cuando se produce un aumento sostenido de islamofobia. Frente a esta situación, la Delegación de Asociaciones Israelitas Argentinas (DAIA) optó hacia fines de 1994 por emitir una declaración pública condenando cualquier acción prejuiciosa en contra de los árabes, lo que permitió el inicio de contactos formales con la Federación de Entidades Argentino Árabes (FEARAB).

Las relaciones de las comunidades judías argentinas no se limitan al mundo árabe, sino que también incluyen a comunidades musulmanas sunnitas y, concretamente, al Centro Islámico de la República Argentina (CIRA), así como a la Iglesia Católica. En estos diálogos tuvo un rol importante el cardenal Bergoglio, actual Papa Francisco, convocando a seminarios y encuentros conjuntos, a partir de su profunda convicción en el diálogo religioso que ha reforzado una vez asumido el Papado: "no puede haber paz sin diálogo". El CIRA y la comunidad Bet El han sido pilares fundamentales del diálogo interreligioso, junto a la Iglesia Católica.

En el contexto de la crisis política, económica y social de 2001, se crearon instancias de diálogo, conocidas como Diálogo Argentino, lideradas por la Iglesia Católica y el cardenal Bergoglio, que convocaron a organizaciones sociales, políticas, educativas, empresariales, sindicales, tendientes a superar la profunda crisis que vivía el país. En el ámbito religioso, estas iniciativas contaban con la representación de las comunidades judías, árabes y musulmanas. Ese mismo año se creó el Instituto del Diálogo Interreligioso por parte del presbítero Guillermo Marcó de la Pastoral Universitaria; el rabino Daniel Goldman, de la Comunidad Bet El; y Omar Abboud, secretario general del CIRA. Los representantes de estas tres religiones establecieron las bases para un diálogo inicial, que permitiera puntos de coincidencia a través de la realización de actividades sociales, culturales, educativas y artísticas, lo que se ha mantenido en el tiempo.

En los funerales del presidente del CIRA, Abel Madel, realizado en 2005, estuvieron presentes el cardenal Bergoglio y las principales autoridades de la AMIA y la DAIA. Ese mismo año, los representantes de las tres religiones monoteístas con presencia en Buenos Aires, suscribieron una declaración conjunta, firmada por el cardenal Jorge Bergoglio; el vicepresidente de la DAIA, León Cohen Bello; el presidente de la AMIA, Luis Grynwald; y el presidente del CIRA, Omal Helal Massud. También fue suscrito por el rabino Daniel Goldman, de la comunidad BetEl, y por Omar Abboud, del CIRA, ambos integrantes fundadores del Instituto del Diálogo arriba mencionado. En este documento se adquirieron los siguientes compromisos: a) educación que fomente el compromiso por la paz y la convivencia; b) profundización del diálogo interreligioso; c) rechazo a cualquier manifestación de violencia; d) creación de una comisión destinada al estudio y prevención de las causas que generan el terrorismo y el fundamentalismo.

Posteriormente, en 2006, convocados por la Secretaría de Culto, dependiente de la Cancillería de Argentina, representantes de las tres confesiones monoteístas firmaron también en Buenos 
Aires una declaración, titulada "La vocación de paz y el diálogo entre las comunidades", la que fue suscrita por el presidente de la AMIA, Luis Grynwald; el presidente del Centro Islámico de la República Argentina (CIRA), Samir Salech; monseñor Horacio Benites Astoul, en representación del Arzobispado de Buenos Aires; el secretario de Culto, embajador Guillermo Oliveri. Parte de la Declaración señala lo siguiente:

En un mundo preocupado por la fragilidad de la paz, la Asociación Mutual Israelita Argentina (AMIA), el Centro Islámico de la República Argentina (CIRA) y el Arzobispado de Buenos Aires, con la convocatoria de la Secretaría de Culto de la Nación, se sienten en la obligación ética de hacer un firme y racional Ilamado a la paz. Aspiramos a que estas palabras sirvan para que cada uno de nosotros, no importa la fe que profese, se proponga reflexionar con profundidad sobre los valores universales que compartimos, como lo viene promoviendo el Instituto del Diálogo Interreligioso. (López, 2006)

El ya mencionado CIRA, junto a la Comunidad Bet-El, y la Cátedra Juan Pablo II de la Universidad Católica de Argentina, organizaron el año 2008 las jornadas de estudio denominadas "El Mediterráneo, puente entre Oriente y Occidente: Las relaciones entre el Cristianismo, el Judaísmo y el Islam", que contaron con la participación, entre otros, del rabino Daniel Goldman de Bet-El y del secretario general del CIRA, Omar Aboud. También resulta importante mencionar el proyecto "Constructores de Puentes", en que participan jóvenes argentinos de 18 a 30 años que profesan las religiones judía, cristiana y musulmana, que tiene por finalidad la búsqueda del mutuo conocimiento y la diversidad. Esta iniciativa, desarrollada en 2008, contó con el patrocinio del Museo Judío de Buenos Aires, la Iniciativa de Religiones Unidas, la Fundación de la Amistad Argentino- Turca y la Embajada de Estados Unidos en Argentina.

Debemos mencionar el rol que han jugado los rabinos Sergio Bergman y Marcelo Polakoff, así como Omar Aboud, ya varias veces mencionado. El primero, liderando la Congregación Israelí de la República Argentina, mantuvo contactos con el CIRA, tendientes a implementar programas de asistencia social a los necesitados, donde Aboud tuvo también un papel protagónico. Es de destacar que ambos representantes religiosos están políticamente unidos al partido Propuesta Republicana (PRO) que preside el intendente de Buenos Aires, Mauricio Macri, Por su parte, el rabino de la comunidad judía de Córdoba, Marcelo Polakoff, mantiene excelentes relaciones con la comunidad musulmana de la ciudad, los dirigentes de ambas comunidades han realizado desde 2007 almuerzos de camaradería en las fiestas de Rosh Hashaná y Ramadán.

En todas estas iniciativas de diálogo interreligioso la iglesia católica ha jugado un rol central, tratando de aunar a las comunidades judías y musulmanas con presencia en Argentina. En enero de 2014 el Papa recibió a los máximos representantes de la DAIA y del Seminario Rabínico Latinoamericano, en tanto que en mayo del mismo año acogió a una delegación del CIRA, todos estos actores comprometidos en el diálogo. El compromiso papal por el diálogo y la paz se ratificó en junio de 2014, con motivo de la visita conjunta de los presidentes de Israel, Shimon Peres, y de la Autoridad Nacional Palestina, Mahmoud Abbas, y una consiguiente oración histórica por la paz palestino-israelí. 
Es importante agregar que estas iniciativas de diálogo que caracterizan a Argentina no sólo han sido reconocidas por el Plan Nacional de la AC elaborado por este país, sino también por el Departamento de Estado de Estados Unidos que en un informe sobre libertad religiosa internacional, elaborado en 2004, da cuenta que la fraternidad y el diálogo religiosos son promovidos por una serie de organizaciones que incluyen la Hermandad Argentina judíacristiana, así como las mencionadas FEARAB y DAIA, que buscan consolidar los encuentros entre musulmanes, judíos, cristianos y árabes (U.S. Department of State, 2004).

\section{CONCLUSIONES}

En el mundo de la posguerra fría, en el marco de la importancia creciente que adquieren los actores transnacionales y la emergencia de nuevos paradigmas para explicar la nueva realidad internacional, destaca el modelo del civilizacionismo, debido al énfasis que otorga a los actores e identidades culturales y civilizacionales como nuevos patrones de explicación de las dinámicas de cooperación y conflicto de las relaciones internacionales.

Dentro del civilizacionismo, existen dos vertientes principales: la del choque civilizacional y la del diálogo civilizacional. La primera coloca el acento en los conflictos que se registran entre civilizaciones distintas, a las que considera como unidades monolíticas y coherentes. La segunda, en cambio, señala un énfasis en el diálogo, en los encuentros, en la AC, considerando las múltiples dimensiones que caracterizan a las civilizaciones.

El aporte de este enfoque de civilizacionismo de diálogo a las relaciones internacionales, es que concibe la importancia de la cultura y la religión como factores determinantes del sistema internacional, superando el paradigma estatocéntrico y aportando con ello al estudio de una realidad internacional de mayor riqueza y complejidad. Busca construir entendimiento y tolerancia entre culturas, pueblos y religiones distintas, a través de un análisis de la realidad internacional centrado en un proceso de construcción de una cultura de paz y diálogo. Para ello concibe a las civilizaciones como unidades que contienen una enorme diversidad en su interior, constatación que explica los conflictos al interior de las civilizaciones, así como también ofrece un espectro de posibilidades de diálogo y cooperación intra e inter civilizaciones.

Este artículo ha explicado los principales puntos teóricos de este enfoque demostrando su validez y aporte a problemas de la realidad internacional, para lo cual, consideramos tres niveles de análisis: global, regional y local, refiriéndonos a los procesos que se dan en el marco de Naciones Unidas, América Latina y el caso argentino, respectivamente.

Al respecto, es posible concluir, en primer lugar, que tanto en los foros globales de diálogo de civilizaciones, así como en las propuestas de AC y en los planes implementados, tanto internacionales como latinoamericanos, el énfasis está marcado en encontrar soluciones a partir de cuatro ejes principales: educación, jóvenes, migraciones, medios de comunicación. 
En segundo lugar, este proceso no es lineal, sino que se retroalimenta constantemente en la trayectoria de lo local a lo global, siendo por lo tanto relevante analizar las experiencias a nivel global, así como las locales, donde el caso argentino demostró su aporte analítico. Además, el diálogo está marcado previamente por experiencias de conflicto: los acontecimientos del 11S en Estados Unidos y $11 \mathrm{M}$ en España fueron un impulso para comenzar a gestar un consenso para el diálogo civilizacional, avanzando desde entonces en un proceso de institucionalización que lleva a la AC. Esta premisa se repite también a nivel local, en cuanto los graves atentados en Argentina impulsan la institucionalización del diálogo entre comunidades distintas. Por lo tanto, el aporte del enfoque aquí analizado es mostrar la viabilidad y las formas posibles para institucionalizar el diálogo civilizacional, aportando a la disminución del conflicto en las relaciones internacionales.

Concretamente, hemos analizado tres procesos que muestran la viabilidad explicativa de este paradigma en la realidad internacional. El primero es la propuesta del presidente iraní, Mohamed Jatami, en la Cumbre de la Conferencia Islámica de 1997, proponiendo un diálogo de civilizaciones, que se concreta el año 2001, con la proclamación del "Año de las Naciones Unidas para el diálogo de la Civilizaciones". El segundo, encabezado por la UNESCO, en base al concepto de diálogo interreligioso, se concreta en noviembre de 2001, con la "Declaración Universal sobre Diversidad Cultural". El tercero comprende los encuentros de AC que se originan el año 2004 bajo la iniciativa de los gobiernos de España y Turquía, que ha dado origen a cinco foros internacionales.

En el ámbito latinoamericano, hay que destacar la presencia de una estrategia regional de AC, la que considera el rol de la región en cuatro grandes tópicos: educación, jóvenes, migraciones, medios de comunicación. En esta línea, desde el año 2009, y desarrollando estos mismos temas, en la región existen tres planes nacionales sobre la AC de México, Brasil y Argentina. El plan brasilero se apoya en la importancia que tiene la política exterior de Brasilia en la mantención de encuentros y foros con otros bloques (IBSA, ASPA, ASA, CPLP) como un eje central de la AC.

El caso argentino resulta emblemático para el estudio del nuevo paradigma. Los ataques de 1992 y 1994, realizados contra la Embajada de Israel y contra la AMIA, respectivamente, en Buenos Aires, son una manifestación importante de antisemitismo y antisionismo y, al mismo tiempo, conllevan a una fuente de diálogo institucionalizado entre comunidades judías, árabes y musulmanas, que continuó en el contexto de la crisis política, social y económica que vivió Argentina hacia fines del 2001.

Por último, es necesario señalar que el paradigma presenta muchas limitaciones, las cuales para algunos tienen atisbos de idealismo por su difícil concreción en la realidad internacional y porque la teoría se adelanta a la práctica. No obstante, puede ser un enfoque útil al análisis internacional. El enfoque del diálogo debería nutrirse y complementarse con otras corrientes, fuentes y escuelas, importantes en estudios internacionales, relevantes en un mundo cada vez más interdependiente y globalizado. Cabe mencionar, entre otros, las investigaciones para la paz, los estudios sobre 
resolución de conflictos, la sociología de las relaciones internacionales, el constructivismo social, la corriente poscolonial, los enfoques sobre integración regional y cooperación internacional, los estudios sobre derechos humanos y derecho internacional humanitario.

\section{REFERENCIAS}

Borradori, G. (2003). Philosophy in a Time of Terror. Dialogues with Jurgen Habermas and Jacques Derrida. Chicago: The University of Chicago Press.

Collier, P. (2004). Development and Conflict. Centre for the Study of African Economies. Oxford: Oxford University. Recuperado de http://www.un.org/esa/documents/Development.and. Conflict2.pdf

Dallmayr, F. (2002). Dialogue among Civilisations: Some Exemplary Voices. Nueva York: Palgrave.

(2009). Justice and Cross-Cultural Dialogue: From Theory to Practice. En. Michális, M. \& Petito, F. (Ed.). Civilizational Dialogue and World Order: The Other Politics of Cultures, Religions, and Civilizations in International Relations. (pp. 29-45). Nueva York: Palgrave Macmillan.

Della, S. (2014). World Jewish Population, 2012. Recuperado de. https://www.jewishvirtuallibrary. org/jsource/Judaism/jewpop.html.

Departamento de Estado de los Estados Unidos de América, Departamento de Estado. (2004). Argentina. International Religious Freedom Report 2004. Recuperado de http://www.state. gov/j/drl/rls/irf/2004/35522.htm

Duffield, M. (2004). Las Nuevas Guerras En El Mundo Global. La Convergencia Entre Desarrollo Y Seguridad. Madrid: Catarata.

Euro-Mediterranean Conference (1995). "Barcelona Declaration" Recuperado de http:// europa.eu/legislation_summaries/external_relations/relations_with_third_coun

Ferguson, N. (2006). Geopolítica. El Choque de Civilizaciones. Letras Libres. 93-95. Recuperado de http://www.letraslibres.com/revista/letrillas/el-choque-de-civilizaciones

Fernández, J. (2008). Choque vs Alianza de Civilizaciones. Intersticios. Revista Sociológica de Pensamiento Crítico. 2(2). 235-240.

Fuentes, I. (2005). Proyecto de Diálogo Interreligioso (documento de Consulta). Recuperado de https://www.google.com.co/url?sa $=t \& r c t=j \& q=\& e s r c=s \&$ source $=$ web $\& c d=1 \& c a d=r j a$ 
\&uact $=8 \&$ ved $=0$ CBsQFjAAahUKEwig67bSw6bIAhXI1B4KHW5bCQA\&url=http\%3A\%2 F\%2Fwww.lacult.org\%2Fdocc\%2FDocumento_Ivette.doc\&usg=AFQjCNFRxT12ydH-1xy BNkWsOuxBkJq9pA\&sig2=2RY8R9uMmn9KzrUIB845ZQ\&bvm=bv.104317490,d.dmo

Fukuyama, F. (2001, noviembre 27). "No hay Choque de Civilizaciones". La Nación. Recuperado de http://www.lanacion.com.ar/354542-no-hay-choque-de-civilizaciones

Gray, C. (1997). Postmodern War: The New Politics of Conflict. Nueva York: The Guilford Press.

Hall, M. \& Jackson, P. (2007). Civilizational Identity: The Production and Reproduction of Civilizations in International Relations. Nueva York: Palgrave.

Holsti, K. (1996). The State, War and the State of War. Cambridge: Cambridge University Press.

Huntington, S. (1993) The Clash of Civilizations? Foreign Affairs. 72(3). 22-49.

(1996). El Choque de Civilizaciones y La Reconfiguracion Del Orden Mundial. Buenos Aires: Paidós.

III Cumbre ASPA: Cinco claves sobre el encuentro con países árabes que se realizará en Lima (2012, septiembre 28). El Comercio. Recuperado de http://elcomercio.pe/mundo/ actualidad/iii-cumbre-aspa-cinco-claves-sobre-encuentro-paises-arabes-que-se-realizaralima-noticia-1475317

International Helsinki Federation for Human Rights. (2005). Intolerance and Discrimination against Muslims in the EU. Development since September 11. Helsinki. Recuperado de http://www.art1.nl/nprd/factsheets/Intolerance\%20against\%20muslims\%20in\%20the\%20 EU\%2003-2005.pdf

Jatami, M. (2012). “2001: Año Del Diálogo Entre Civilizaciones”. El Muecín. Recuperado de http://www.organizacionislam.org.ar/muecin52/dialogo.htm

Kaldor, M. (2001). Las Nuevas Guerras. Violencia Organizada En La Era Global. Barcelona: Tusquets. (94). 11-20.

Kalyvas, S. (2001). "New" and "old" Civil Wars. A Valid Distinction? World Politics. (54). 99-118.

Katzenstein, P. (2009). Civilizations in World Politics: Plural and Pluralist Perspectives. Nueva York: Routledge. 
Lewis, B. (1990). The Roots of Muslim Rage. The Atlantic Monthly. 266(3). 47 - 60.

López, R. (2002). ¿Hay Choque de Civilizaciones? Reis. (97). 229.

(2013). "Argentina da ejemplo de convivencia con una carta interreligiosa". Webislam. Recuperado de. http://www.webislam.com/noticias/47074-argentina_da_ejemplo_de_ convivencia_con_una_carta_interreligiosa.html

Marchetti, R. (2009). Civilizationism and the Political Debate on Globalization. En. Michael, M. \& Petito, F. (Ed.) Civilizational Dialogue and World Order: The Other Politics of Cultures, Religions, and Civilizations in International Relations. (pp. 93 - 110). Nueva York: Palgrave Macmillan.

Melander, E.; Öberg, M. \& Hall, J. (2006). Are "new Wars" More Atrocious? Battle Severity, Civilians Killed and Forced Migration before and after the End of the Cold War. European Journal of International Relations. 15(3). 505-536.

Merinero, M. (2002). Diálogo de Civilizaciones Oriente-Occidente. Aporte Al Entendimiento Internacional. Madrid: Biblioteca Nueva, Universidad de Extremadura.

Michael, M. \& Petito, F. (2009). Imperial Monologue or Civilizational or Civilizational Dialogue? En. Michael, M. \& Petito, F. (Ed.) Civilizational Dialogue and World Order: The Other Politics of Cultures, Religions, and Civilizations in International Relations. (pp. 3 - 25). Nueva York. Palgrave Macmillan.

Ministerio de Relaciones Exteriores de la República Argentina . (2010). Alianza de Civilizaciones. República Argentina. Plan Nacional de Acción. Recuperado de http://www. cancilleria.gov.ar/portal/alianza/docs/plan_acc

Ministerio de Relaciones Exteriores de Brasil. (2014). Alliance of Civilizations. National Plan of Brazil. Recuperado de: http://www.unaoc.org/wp-content/uploads/Brazil-NationalStrategy1.pdf

Münkler, H. (2003). The Wars of the 21st Century. International Review of He Red Cross 85(849). 7-22.

Petito, F. (2009). Dialogue of Civilizations as an Alternative Model for World Order. En. Michael, M. \& Petito, F. (Ed.) Civilizational Dialogue and World Order: The Other Politics of Cultures, Religions, and Civilizations in International Relations. (pp. 48 - 67). Nueva York: Palgrave Macmillan.

Said, E. (2002). La Guerra Y El Choque de Las Ignorancias. Educere. 6(18). 221-224. 
Salter, M. (2002). Barbarians and Civilization in International Relations. Londres: Pluto Press.

Secretaría de Relaciones Exteriores de México (2010). Plan Nacional de Diálogo Intercultural de México Para La Alianza de Civilizaciones. México: Secretaría de Relaciones Exteriores de México.

Organización de las Naciones Unidas para la Educación, la Ciencia y la Cultura, UNESCO. (2002). Declaración Universal de la UNESCO Sobre La Diversidad Cultural. Recuperado de http:// portal.unesco.org/es/ev.php-URL_ID =13179\&URL_DO=DO_TOPIC\&URL_SECTION=201. html

Organización de las Naciones Unidas, ONU. (2009). Resolución adoptada por la Asamblea General 69/312. The Alliance of Civilizations. Recuperado de http://www.un.org/en/ga/ search/view_doc.asp?symbol=A/RES/69/312

Organización Islámica para América Latina (2014). Los Musulmanes En Latinoamérica. Recuperado de http://www.islamerica.org.ar/organizacion/musulmanes_latinoamerica. html

Ramadan, T. (2014). Alliance of Civilizations? Recuperado de http://tariqramadan.com/ english/2011/12/17/alliance-of-civilizations/

Sen, A. (2006, mayo 24). “¿Qué Choque de Civilizaciones?” Rebelión. Recuperado de http:// www.rebelion.org/noticia.php?id=31950

Wacquant, L. (2000). The New "peculiar Institution": On the prison as surrogate Ghetto. Theoretical Criminology. 4(4). 377 - 389.

Wallerstein, I. (1997). El Futuro de La Civilización Capitalista. Barcelona: Icaria Editorial. 